\title{
Surveillance of adverse events following immunization: 10 years' experience in Oman
}

S. Al Awaidy, ${ }^{1}$ S. Bawikar, ${ }^{1}$ K.P. Prakash, ${ }^{2}$ B. Al Rawahi ${ }^{7}$ and A.J. Mohammed ${ }^{2}$

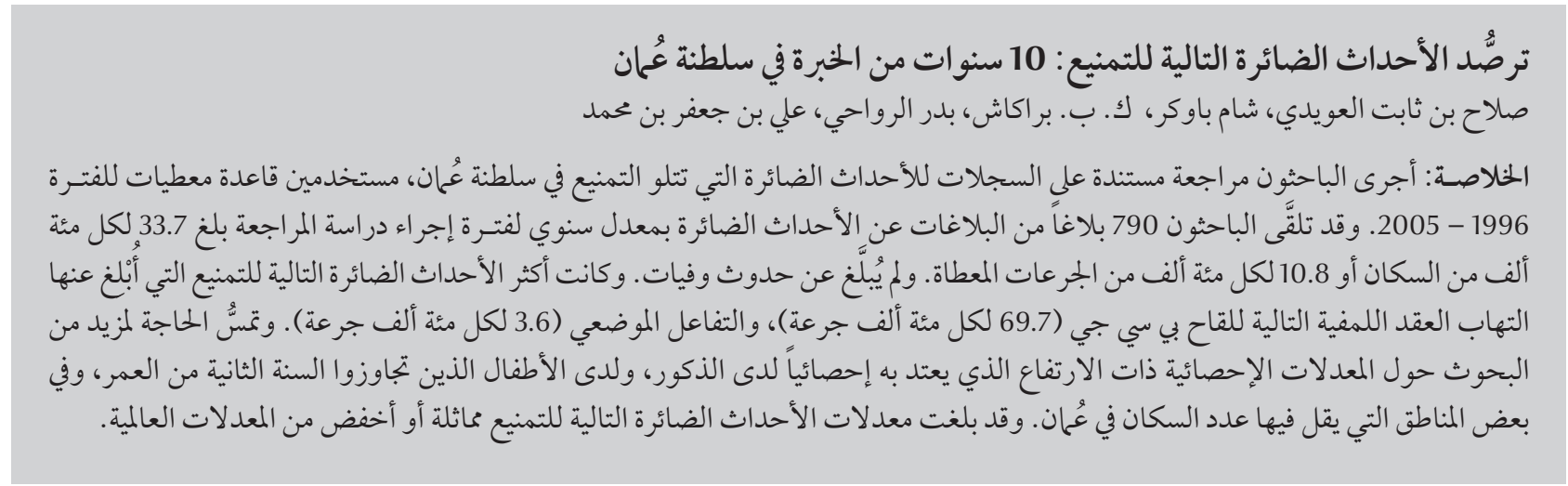

ABSTRACT A descriptive record-based review of adverse events following immunization (AEFI) was carried out in Oman using the national database for the period 1996-2005. A total of 790 adverse event reports were received with an annual rate during the review period of 33.7 per 100000 population or 10.8 per 100000 doses administered. There were no reported deaths. The most frequently reported AEFI were BCG adenitis (69.7 per 100000 doses) and local reactions (3.6 per 100000 doses respectively). The statistically significant higher rates among males, in children aged $>2$ years and in some sparsely populated regions of Oman need further research. AEFI rates in Oman were similar or below the international averages

\section{Surveillance des manifestations postvaccinales indésirables : dix ans d'expérience à Oman}

RÉSUMÉ Une analyse descriptive basée sur les enregistrements de manifestations postvaccinales indésirables $(\mathrm{MAPI})$ a été réalisée à Oman à l'aide des bases de données nationales pour la période comprise entre 1996 et 2005. Un total de 790 rapports de manifestations indésirables a été reçu avec un taux annuel au cours de la période de 33,7 pour 100000 patients ou 10,8 pour 100000 doses administrées. Aucun décès n'a été signalé. Les MAPI les plus fréquemment rapportées étaient la lymphadénite associée au BCG (69,7 pour 100000 doses) et des réactions locales (3,6 pour 100000 doses). La supériorité statistiquement significative des taux d'incidence observés chez les hommes, chez les enfants de plus de deux ans, et dans quelques régions peu peuplées d'Oman, nécessite des recherches supplémentaires. Les taux de MAPI relevés à Oman étaient semblables ou inférieurs aux moyennes internationales.

'Department of Communicable Disease Surveillance and Control, Ministry of Health, Muscat, Oman (Correspondence to S. Al Awaidy: salah,awaidy@gmail.com).

${ }^{2}$ Directorate General of Health Services, Ministry of Health, Muscat, Oman.

Received: 27/06/07; accepted: 19/03/08 


\section{Introduction}

Vaccines, in addition to the desired outcome of provoking an immune response, may also produce some degree of unwanted (adverse) reactions. While the great majority of these are minor and harmless, a very small number are serious and potentially life-threatening. To increase acceptance of immunization and to improve the quality of services, the surveillance of adverse events following immunization (AEFI) must become an integral part of any national immunization programme.

The primary objectives of AEFI surveillance are: to monitor the trend of known adverse events; detect new, unusual or rare vaccine adverse events; determine patient risk factors for particular types of adverse events; identify vaccine lots with increased numbers or types of reported adverse events; and monitor adverse events after the marketing of newly introduced vaccines. The surveillance should also aim to identify preventable events and to take appropriate action wherever possible. A passive AEFI surveillance system seeks voluntary reports from patients visiting health care facilities and reports from health care professionals of any clinical event that occurs after vaccination, even if the reporter cannot be certain that the event was caused by the vaccine. Hence, the limitations of passive AEFI include wide variability in reporting standards, reporter bias and probably under-reporting of events.

In $200153 \%$ of all World Health Organization (WHO) member countries reported having a national AEFI monitoring system, an increase from $45 \%$ in 2000 . The proportion among the countries of the Eastern Mediterranean Region (EMR) was 59\% in the year 2001 [1]. In Oman, as of 2005, 10 antigens are included in the Expanded Programme on Immunization (EPI) for infants. Through an efficiently managed programme, over $95 \%$ coverage has been achieved for all vaccines since
1989. A passive AEFI surveillance programme was launched in 1996 to address vaccine safety concerns. This paper presents an analysis of the data on AEFI of childhood vaccinations reported over the 10-year period 1996-2005.

\section{Methods}

This was a record-based descriptive study of reported AEFI events in children aged $<6$ years where the date of vaccination or onset of the adverse event occurred between January 1996 and December 2005.

The Oman EPI manual, with its standard operating procedures, was published by the Ministry of Health in 2003 and includes a section on AEFI [2]. As a Ministry of Health policy, all AEFI are required to be notified within 24 hours. All government institutions at all levels of health care offering immunization services and 16 vaccine qualified private clinics were included in the surveillance network. The EPI staff in the health care facilities were trained in the identification of events and the reporting protocol, in addition to receiving ongoing in-service training. During periodic national supervisory visits, EPI staff were updated on new and/or revised policies.

An AEFI was defined as any adverse event that occurred after a vaccination, which might be related to the vaccine itself or to its handling or administration [2-4]. A serious adverse event following vaccination was defined as when a patient died, experienced a life-threatening illness or required hospitalization or when the condition resulted in permanent disability.

All medical events (usual or unusual, minor or serious) related to vaccines, for which medical care was sought, were considered, including all cases of BCG lymphadenitis; injection site abscesses; and deaths, hospitalizations and other severe or unusual medical incidents that were thought by health workers or the patients, to be related to immunization. Mild episodes of fever after pentavalent vaccine or mild local pain and swelling were not required to be reported. However, reporting of vaccine sensitivity events was given precedence.

The following vaccines were studied: bacille Calmette-Guérin (BCG), hepatitis B virus (HBV), Haemophilus influenzae type B (Hib), measles, rubella, measles-rubella (MR), measles-mumps-rubella (MMR), oral polio vaccine (OPV), diphtheriatetanus (DT), tetanus toxoid (TT), diphtheria-tetanus-pertussis (DTP) and pentavalent (DTP-Hib-HBV).

The data were collected by the EPI section of the Ministry of Health and compiled and analysed on a monthly basis at the national level. A modified version of the WHO recommended reporting form with standard case definitions was adopted [3]. Regular feedback was given through monthly reports for follow-up and action. A compiled report was submitted to the WHO Regional Office for the Eastern Mediterranean at the end of every quarter. All serious events as well as clusters of events were investigated within a reasonable time frame. In specific situations, e.g. a clustering of AEFI events following MR vaccination observed in October 1995, a detailed epidemiological investigation was conducted [5]. A serious adverse event following DTP vaccine administration in the year 2000 was also thoroughly investigated [6].

The data were compiled in Microsoft Excel format and data analysis was performed using SPSS, version 9.0. Average annual population-based rates of AEFI were calculated for different variables using the 2003 census data. Dose-related AEFI rates were calculated by using the number of doses administered from the national database as the denominator. Relative risk (RR) and $95 \%$ confidence intervals (CI) were calculated using the national average as the reference. A $P$ value of $<0.05$ was considered as statistically significant. 


\section{Results}

\section{Overall reporting rates (1996- 2005)}

A total of 790 AEFI reports were received in the period 1996-2005 from a total of 7292640 vaccine doses administered to children aged $<6$ years. The overall reporting rate for this period was therefore 10.8 per 100000 doses administered or 33.7 per 100000 population (based on the 2003 census figure for the population of Oman of 2340815 ).

\section{Reporting rates by sex, age and region (1998-2005)}

The data by sex, age and region were available as an electronic database only for the period 1998-2005 (Table 1). There were 657 reports over this period, a reporting rate of 10.7 per 100000 doses administered.
A higher proportion of AEFI reports were among males than females (59.4\% versus $40.6 \%$ ) and the reporting rate per 100000 doses administered was also significantly higher among males than females (12.6 versus 8.8) $(\mathrm{RR}=1.1$, 95\% CI: $1.0-1.3, P<0.05)$.

The highest proportion of adverse events was among children aged $<1$ year $(80.5 \%)$, compared with those aged $1-2$ years $(12.6 \%)$ and $>2$ years (6.8\%). However, the reporting rate per 100000 doses administered was significantly higher among those aged > 2 years (402.7) compared with the national average (10.7) $(\mathrm{RR}=37.5,95 \%$ CI: 22.7-50.7, $P<0.05)$ and compared with those aged $<1$ year (10.9) and 1-2 years (6.6).

The distribution of AEFI reports by province (regions/governorates) for this period indicated that 4 regions had higher rates of adverse event reports per
100000 doses administered compared with the national average: Dhahira (11.9), Dakhliyah (14.5), North Sharqiyah (41.3) and Musandam (69.6). However, only 3 regions (Dakhliyah, North Sharqiyah and Musandam) had statistically significant higher rates compared with the national average for this period (10.7) (Table 1).

\section{Reporting rates by type of vaccine (1996-2005)}

Figure 1 shows the number of reports for each vaccine for the full reporting period (1996-2005). BCG vaccine was responsible for the highest number of AEFI reports (326), both as a percentage of all 790 AEFI reports (41.3\%) and as the rate per 10000 doses of BCG administered (69.7). DTP was the second most common vaccine associated with AEFI reports (296); $37.5 \%$ of all reports or 15.3 per 10000 doses of DTP administered. Pentavalent vaccine was the

\begin{tabular}{|c|c|c|c|c|c|c|}
\hline \multirow[t]{2}{*}{ Variable } & \multicolumn{2}{|c|}{ AEFI reports } & \multirow{2}{*}{$\begin{array}{l}\text { AEFI per } 100000 \\
\text { population }^{\mathrm{a}}\end{array}$} & \multirow{2}{*}{$\begin{array}{l}\text { No. of doses } \\
\text { given }\end{array}$} & \multirow{2}{*}{$\begin{array}{c}\text { AEFI per } \\
100000 \text { doses }\end{array}$} & \multirow[t]{2}{*}{$\operatorname{RR}(95 \% \mathrm{Cl})$} \\
\hline & No. & $\%$ & & & & \\
\hline \multicolumn{7}{|l|}{ Age (years) } \\
\hline$<1$ & 529 & 80.5 & - & 4851341 & 10.9 & $1.0(0.09-0.13)$ \\
\hline $1-2$ & 83 & 12.6 & - & 1262031 & 6.6 & $0.6(0.4-0.7)$ \\
\hline$>2$ & 45 & 6.8 & - & 11175 & 402.7 & $37.5(29.6-54.2)^{*}$ \\
\hline \multicolumn{7}{|l|}{ Sex } \\
\hline Male & 390 & 59.4 & 29.7 & 3104094 & 12.6 & $1.1(1.2-1.7)^{*}$ \\
\hline Female & 267 & 40.6 & 26.0 & 3020453 & 8.8 & $0.8(0.6-0.8)$ \\
\hline \multicolumn{7}{|l|}{ Region } \\
\hline Musandam & 43 & 6.6 & 151.5 & 61758 & 69.6 & $6.4(5.1-9.4)^{*}$ \\
\hline North Sharqiyah & 187 & 28.5 & 133.5 & 452698 & 41.3 & $3.8(4.2-5.9)^{*}$ \\
\hline Dakhliyah & 116 & 17.7 & 43.4 & 800272 & 14.5 & $1.3(1.2-1.7)^{*}$ \\
\hline Dhahira & 57 & 8.7 & 27.5 & 478469 & 11.9 & $1.1(0.8-1.5)$ \\
\hline Wustah & 5 & 0.8 & 21.8 & 49122 & 10.2 & $0.9(0.4-2.3)$ \\
\hline North Batinah & 100 & 15.2 & 24.5 & 1069951 & 9.3 & $0.8(0.7-1.1)$ \\
\hline Dhofar & 37 & 5.6 & 17.1 & 544387 & 6.8 & $0.6(0.4-0.8)$ \\
\hline South Batinah & 40 & 6.1 & 16.4 & 682381 & 5.9 & $0.5(0.4-0.7)$ \\
\hline Muscat & 61 & 9.1 & 9.7 & 1476834 & 4.1 & $0.4(0.2-0.4)$ \\
\hline South Sharqiyah & 11 & 1.7 & 6.3 & 508675 & 2.2 & $0.2(0.1-0.3)$ \\
\hline Total & 657 & 100.0 & 28.1 & 6124547 & 10.7 & Reference \\
\hline
\end{tabular}

${ }^{a}$ Based on population of Oman (2003 census);

${ }^{*} P<0.05$.

- = data not available $; R R=$ relative risk; $C I=$ confidence interval . 


\begin{tabular}{|c|c|c|c|}
\hline \multirow[t]{2}{*}{ Type of AEFI } & \multicolumn{2}{|c|}{ AEFI reports } & \multirow{2}{*}{$\begin{array}{c}\text { AEFI per } \\
100000 \text { doses }^{\mathrm{a}}\end{array}$} \\
\hline & No. & $\%$ & \\
\hline Adenitis (BCG) & 326 & 41.3 & 69.7 \\
\hline Local reactions & 260 & 32.9 & 3.8 \\
\hline Systemic reactions & 131 & 16.6 & 1.9 \\
\hline Injection abscess & 73 & 9.2 & 1.1 \\
\hline Total & 790 & 100.0 & 10.8 \\
\hline
\end{tabular}

${ }^{a}$ Number of doses given was 7292640 except for BCG adenitis (467 555). $B C G=$ bacille Calmette-Guérin .

third most commonly associated with adverse events. There were 71 reports (21.7 per 100000 doses administered) during the period when it was used (2003-05). There were 17 reports for MMR (3.6 per 100000 doses administered), 14 for HBV (1.5 per 100000 doses), 14 for measles (2.9 per 100000 doses) and 4 for Hib (2.3 per 100000 doses). OPV was not associated with any adverse events.

\section{Reporting rates by type of adverse events (1996-2005)}

Table 2 shows the number of AEFI reports by type of reaction for period 1996-2005. The most common adverse events were BCG adenitis, accounting for $41.3 \%$ (326/790) reports over the 10-year review period, followed by local reactions, such as severe pain and/or swelling, accounting for 32.9\% (260/790). Injection abscess was reported in $9.2 \%$ of AEFI $(73 / 790)$. The dose-related rates showed the same pattern: the 2 most commonly reported adverse reactions were BCG adenitis (69.7 per 100000 BCG doses) and local reactions (3.6 per 100000 doses of allvaccines). The more serious reactions were rarer: systemic reactions (1.8) and injection abscess (1.0). BCG adenitis was a significantly more common event after vaccination when compared with adverse events following other vaccines $(\mathrm{RR}=10.3,95 \% \mathrm{CI}$ : 9.5-12.6, $P<0.001)$.

\section{Types of events caused by vaccines}

Although there were a high number of adverse events associated with pentavalent and DTP vaccine, a majority of them were local minor events (Table 3). For DTP, local reactions, systemic reactions and injection abscess occurred in $57.5 \%, 24.6 \%$ and $17.9 \%$ of reactions respectively. For the pentavalent vaccine $62.1 \%$ of the events were local reactions and $22.3 \%$ systemic reactions. Of reports for measles vaccine, $88.9 \%$ were systemic reactions such as fever and for MMR, $52.9 \%$ were systemic reactions.

The rate of BCG injection abscess was $1.2 \%$ or 0.8 per 100000 doses administered.

There were no serious or systemic events reported for BCG, Hib or OPV vaccines. However, serious adverse events associated with measles/MMR (3.4\%) included convulsions (1 case), febrile convulsions (1) and anaphylactic reactions (5) and with DTP were convulsions (8 cases), febrile convulsions (8), anaphylaxis (2) and unsteady gait (2).

\section{Comparisons with other countries}

Tables 4 and 5 compare the rates of AEFI reports in the USA, Canada and Australia with those found in the present study in Oman. The data show that the AEFI figures in Oman compare favourably with those in such developed countries.

\section{Discussion}

In Oman, the Ministry of Health policy dictates mandatory notification of all vaccine-related adverse events within 24 hours [4]. This paper provides an overview of all reported AEFI during the period 1996-2005. Of course not all these events were necessarily caused by vaccines. AEFI surveillance is complex, because the association between the reported exposure(s) and outcome(s) is temporal but not always causal. Inadequate or misleading information may have an adverse impact on the analysis and interpretation of AEFI surveillance data. Identification, detection, prevention and appropriate reporting of AEFI are therefore essential in ensuring the safety of vaccinations

During 1996-2005, we received 790 reports out of over 7 million doses of vaccines that were administered. The overall rate for the reported vaccine types was 10.8 per 100000 doses administered to children aged $<6$ years, which is similar to the rates in USA (11.4 per 100000 doses) [7] and Australia (11.8 per 100000 doses for children aged $<7$ years) [8]. Under-reporting could be a factor due to the passive reporting system [9]. Symptomatic local reactions and fever are reported to occur in about $10 \%$ of all vaccine recipients, except for DTP and $T$ boosters, in which they affect about 50\% [10].

The number of adverse events in males was significantly higher than in females. Conversely, in other studies there were more events in females or only minimal differences between the sexes $[7,8,11]$. The highest proportion of AEFI reports was among children aged $<1$ year $(80.5 \%)$, which reflects the fact that this is the age group receiving the greatest numbers of vaccinations. These proportions were lower in the USA (18.1\%) [7] and Canada (approximately 54\%) in the age group $<1$ year [11]. These variations in rates between countries may be largely due to different vaccines and regimens in 


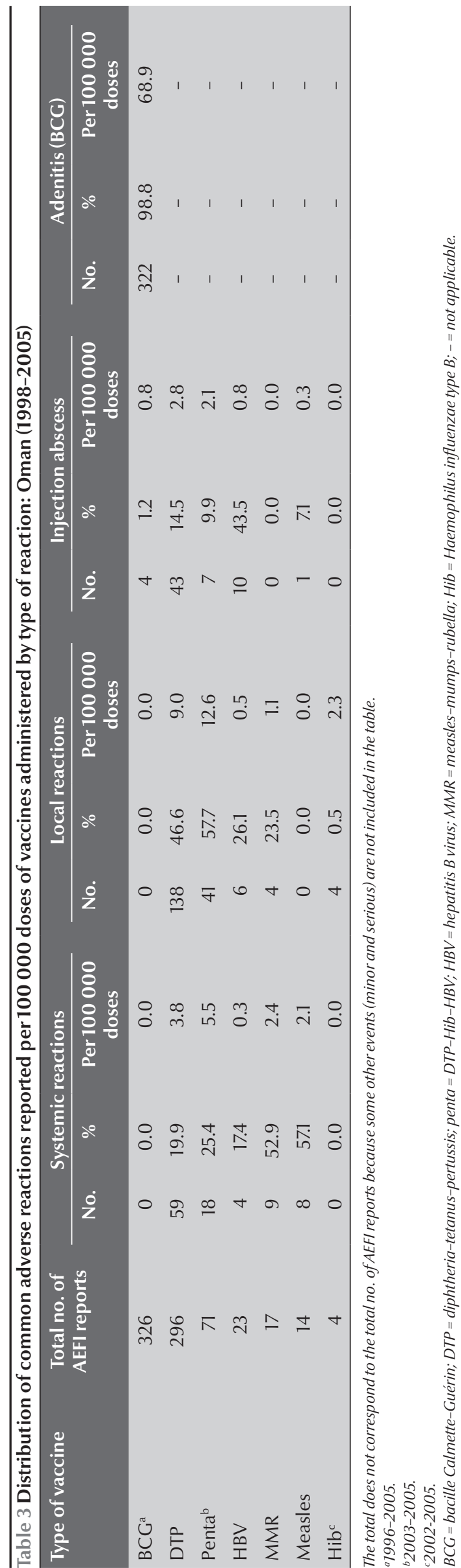

vogue. It was notable that the dose-related rates were much higher among children aged $>2$ years compared with those aged $<1$ and $1-2$ years in our study.

Comparable to reports from the USA, Australia and Canada, the AEFI reporting rates varied among difference provinces in Oman $[7,8,11]$. Provincial variations exist for several reasons that may not be related to actual safety issues. These include variations in vaccination delivery (public or private), reporting rates, staff motivation and special vaccination programmes. It is worth noting that, for reasons unknown, in all these above-mentioned studies including our study, the least populated provinces had higher rates of AEFI. The significantly higher rates of AEFI in males, among children aged $>2$ years and in some regions in Oman needs further investigation.

The most common adverse events were BCG adenitis (41.3\%) followed by local reactions (32.9\%). Local reactions such as fever, injection-site hypersensitivity or oedema, rash, injection-site oedema and vasodilatation were reported in $25.8 \%, 15.8 \%, 10.8 \%$, $11.0 \%$, and $10.8 \%$ of cases respectively in the Omani vaccine adverse event reporting system reports [5]. In Canada, the 3 most commonly reported adverse events were local reactions ( $32.4 \%$ of $3625)$, allergic reactions including rash (31.7\%) and fever (23\%) [11]. In contrast, the rate of serious adverse events in Oman was less $(3.4 \%)$ compared with the data published from the USA in 2003 (14.2\%) [7]. Two other studies found that nonserious reaction rates of $59 \%$ and $48 \%$ and serious reactions rates of $9 \%$ and $1 \%$ of all reactions respectively $[8,11]$.

Injection abscess was reported in $9.2 \%$ of cases (1.0 per 100 000 doses administered). Local reactions are generally common in aluminium-containing vaccines. For BCG vaccine, an injection abscess may result from subcutaneous rather than intradermal delivery of vaccine [3]. The rate of BCG injection abscess was 0.8 per 100000 doses administered. A higher rate of $43.6 \%$ for lymphadenitis and suppurative lymphadenitis (4 cases per 100000 ) was reported from Poland [12]. In the West Indies it was concluded that it was related to the Pasteur strain used in the vaccine in Jamaica [13]. In South Africa, adverse events occurred in $3.1 \%$ of neonates, and a higher proportion of these presented with extranodal injection-site abscesses (41.0\%) and lymphadenopathy (18.0\%) compared with our results [14]. Similarly, in Sweden, regional lymphoglandular swellings ( 1.9 per 1000 vaccinated children) and/or abscesses (1.4 per 1000 vaccinated children) were most commonly reported [15].

After BCG, DTP was the second most common vaccine associated with adverse events. Local reactions, systemic reactions and injection abscess occurred in 46.6\%, 19.9\% and $14.5 \%$ of the cases respectively, which is within the expected rates of 50\%-80\% [9].

Pentavalent vaccine was the third most commonly associated with adverse events. There were 71 reports (21.7 per 100000 doses administered) during the $2003-05$ period. Approximately $58 \%$ of the events were local reactions followed by systemic reactions 


\begin{tabular}{|c|c|c|c|c|c|}
\hline \multirow[t]{3}{*}{ Vaccine } & Oman & USA & Australia & Oman & Canada \\
\hline & $1996-2005^{a}$ & $1991-2001^{b}$ & $2000-2002^{c}$ & $1996-2005^{a}$ & $1993-1997^{d}$ \\
\hline & $\begin{array}{c}\text { AEFI per } 100000 \\
\text { doses }\end{array}$ & $\begin{array}{c}\text { AEFI per } 100000 \\
\text { doses }\end{array}$ & $\begin{array}{c}\text { AEFI per } 100000 \\
\text { doses }\end{array}$ & $\begin{array}{l}\text { No. of AEFI } \\
\text { reports received }\end{array}$ & $\begin{array}{l}\text { No. of AEFI } \\
\text { reports received }\end{array}$ \\
\hline BCG & 69.7 & - & - & 326 & 170 \\
\hline DTP & 15.3 & 26.2 & 42.3 & 296 & 4310 \\
\hline DTP-HBV & - & - & 25.2 & - & - \\
\hline $\mathrm{Hib}$ & 2.3 & 18.1 & 23.1 & 4 & 10487 \\
\hline Hib-HBV & - & 12.9 & 12.1 & - & - \\
\hline Measles & 2.9 & 12.0 & - & 14 & 1244 \\
\hline MMR & 3.6 & 16.3 & 26.6 & 17 & 2188 \\
\hline HBV & 1.5 & 11.8 & 10.4 & 23 & 1961 \\
\hline Penta & 21.7 & - & - & 71 & - \\
\hline
\end{tabular}

Sources: ${ }^{a}$ Current study; $[7] ;$; [8]; ${ }^{d}[11]$.

USA = United States of America; $B C G=$ bacille Calmette-Guérin; DTP=diphtheria-tetanus-pertussis; HBV= hepatitis B virus; Hib= Haemophilus influenzae type B; $M M R=$ measles - mumps - rubella $;$ penta $=D T P-H i b-H B V ;-=$ data not available .

(25\%). In the Ghanaian safety study on pentavalent vaccine (September 2003 to December 2004), 28.3\% of the children attended the study clinic with suspect AEFI including minor and/or unrelated events [16].

Measles and MMR were most commonly associated with systemic reactions such as fever than with local reactions. The MMR AEFI rate was 3.6 per 100000 doses administered. Fever and rash are considered the most common reactions associated with
MMR either in combination or used individually [10]. In the MMR campaign in Australia, the overall rate of adverse events was 5.24 per 100000 doses administered [17].

Similar to a $\mathrm{WHO}$ report on vaccine safety, OPV was not associated with any adverse events [9]. Likewise serious adverse events are uncommon with $\mathrm{HBV}$ and Hib vaccines which in our study produced fewer local/systemic reactions than other vaccines [9]. Measles/MMR and DTP vaccines were associated with a few serious adverse events, i.e. convulsions, febrile convulsions, anaphylaxis and unsteady gait. In the WHO report convulsions accounted for 1 in 12500 doses administered, hypotonic-hyporesponsive episodes 1 per 1750 doses, while anaphylaxis was rare (2 per 100000 doses) [9].

The comparison of the Omani AEFI data with other studies around the world should be interpreted with prudence since the vaccines administered, immunization programmes, AEFI reporting

\begin{tabular}{|c|c|c|c|c|}
\hline \multirow[t]{2}{*}{ Year } & \multicolumn{2}{|c|}{ Oman [current study] ${ }^{\mathrm{a}}$} & \multirow{2}{*}{$\begin{array}{c}\text { Canada }^{\mathrm{b}} \\
\text { AEFI per } 100000 \text { net } \\
\text { distribution doses }\end{array}$} & \multirow{2}{*}{$\begin{array}{c}\text { Australia } \\
\text { AEFI per } 100000 \text { doses } \\
\text { distributed/administered }\end{array}$} \\
\hline & $\begin{array}{l}\text { Total no. of AEFI } \\
\text { reports }\end{array}$ & $\begin{array}{l}\text { AEFI per } 100000 \text { doses } \\
\text { administered }\end{array}$ & & \\
\hline 1996 & 52 & 8.8 & 33 & - \\
\hline 1997 & 81 & 14.0 & 34 & - \\
\hline 1998 & 68 & 8.8 & 27 & - \\
\hline 1999 & 95 & 12.5 & 19 & - \\
\hline 2000 & 105 & 14.0 & 26 & \\
\hline 2001 & 82 & 11.0 & 28 & $19.8^{\mathrm{c}, \mathrm{g}}$ \\
\hline 2002 & 86 & 10.0 & 23 & \\
\hline 2003 & 72 & 8.5 & 16 & $14.6^{\mathrm{d}, \mathrm{h}}$ \\
\hline 2004 & 73 & 10.6 & 17 & $11.8^{\mathrm{e}}$ \\
\hline 2005 & 76 & 11.0 & - & $11.0^{f}$ \\
\hline
\end{tabular}

Sources: ${ }^{a}$ Current study; $b$ [18]; c[19]; d[20]; [21]; f[22].

gPeriod: 01/2000-09/2002; ${ }^{h} 70 / 2002-12 / 2003$. 
system and presentation of data may vary considerably.

\section{Conclusion}

It can be concluded that the AEFI surveillance in Oman is of a high standard as the reported rates are similar or below the international averages or expected rates. Surveillance of AEFI is always an integral component of a national immunization programme. As with any passive surveillance systems, underreporting is likely. Continued efforts on the part of immunization service providers and public health practitioners are essential to strengthen and sustain the quality of AEFI surveillance in Oman. In general, addressing vaccine safety issues effectively preserves the integrity of the immunization programme and avoids undue concerns in the community.

\section{Acknowledgements}

We are grateful to all the EPI doctors and staff and regional EPI supervisors involved in the reporting of the adverse events without whom this analysis would not have been possible.

\section{References}

1. Global status of immunization safety: a report based on the WHO/UNICEF Joint Reporting Form. Weekly epidemiology record, 2003, 78(7):44-7.

2. Mansoor $\mathrm{O}$ et al. Immunization safety surveillance: Guidelines for managers of immunization programmes on reporting and investigating adverse events following immunization. Manila, World Health Organization Regional Office for the Western Pacific, 1999

3. Surveillance of adverse events following immunization, field guide for managers of immunization programmes. Geneva, World Health Organization, 1997.

4. The milestones of EPI in Oman. Manual on expanded program on immunization, 3rd ed. Muscat, Oman, Ministry of Health, 2003:1-62

5. Ministry of Health, Oman. Hypersensitivity reactions reported with MR vaccine. Community health and disease surveillance newsletter, 1995, 4(3):7-8.

6. Ministry of Health, Oman. An unusual presentation of AEFI. Com munity health and disease surveillance newsletter, 2000, 9(1):10.

7. Centers for Disease Control and Prevention (CDC). Surveillance for Safety after Immunization: Vaccine Adverse Event Reporting System (VAERS)-United States, 1991-2001. Morbidity and mortality weekly report, 2003, 52(1):1-24.

8. Lawrence $G$ et al. Surveillance of adverse events following immunization in Australia 2004. Communicable diseases intelligence, 2005, 29(3):248-62.

9. Supplementary information on vaccine safety, Part 2: Background rates of adverse events following immunization. Geneva, World Health Organization, 2000.

10. Stratton KR, Howe CJ, Johnston RB. Research strategies for assessing adverse events associated with vaccines: a workshop sum mary. Washington DC, National Academy Press, 1994.

11. National Report (interim) on Immunization Vaccine Safety Issues and Surveillance 1998. Reported adverse events temporarily associated with vaccine administration in Canada: 1993 to 1997 summary tables. Journal of the Canadian Pediatric Society, 1998, 4 (Suppl C).
12. Szczuka I. [Adverse event after BCG vaccination in Poland in the years 1994-1997]. Pneumonologia i alergologia polska, 1999, 67(5-6):208-16 [in Polish].

13. Praveen $\mathrm{KN}$ et al. Outbreak of Bacillus Calmette-Guerin associated lymphadenitis and abscesses in Jamaican children. Pediatric infectious disease journal, 1990, 9(12):890-3.

14. Prakash MJ et al. Safety of the intra-dermal Copenhagen 1331 BCG vaccine in neonates in Durban, South Africa. Bulletin of World Health Organization, 2001, 79(4):337-43.

15. Romanus $\vee$ et al. Adverse reactions in healthy and immunocompromised children under six years of age vaccinated with the Danish BCG vaccine, strain Copenhagen 1331: implications for the vaccination policy in Sweden. Acta paediatrica, 1993, 82(12):1043-52.

16. Dodoo AN et al. Safety monitoring of a new pentavalent vaccine in the expanded programme on immunisation in Ghana. Drug safety, 2007, 30(4):347-56

17. D'Souza RM et al Adverse events following immunisation associated with the 1998 Australian Measles Control Campaign. Communicable diseases intelligence, 2000, 24(2):27-33.

18. Canadian national report on immunization. Canada communicable disease report, 2006, 32(Suppl. 3):1-44.

19. Lawrence $\mathrm{G}$ et al. Adverse events following immunisation: Australia 2000-2002. Communicable diseases intelligence, 2003, 27:307-23.

20. Lawrence G et al. Surveillance of adverse events following immunisation: Australia 2002-2003. Communicable diseases intelligence, 2004, 28:324-38.

21. Lawrence G et al. Surveillance of adverse events following immunization in Australia 2004. Communicable diseases intelligence, 2005, 29:248-62.

22. Lawrence G et al. Surveillance of adverse events following immunisation: Australia 2005. Communicable diseases intelligence, 2006, 30:319-33. 